'División de Enfermedad Cardiovasculares, Facultad de Medicina, Pontificia Universidad Católica de Chile. Santiago, Chile.

${ }^{2}$ Advanced Center for Chronic

Disease (ACCDiS), Facultad de Medicina, Pontificia Universidad Católica de Chile. Santiago, Chile. ${ }^{3}$ Departamento de Hematología y Oncología, Pontificia Universidad Católica de Chile. Santiago, Chile.

${ }^{4}$ Servicio de Laboratorio Clínico, Pontificia Universidad Católica de Chile. Santiago, Chile. ${ }^{5}$ Advanced Center for Chronic Diseases (ACCDiS) y Centro de Estudios Moleculares de la Célula

(CEMC), Facultad de Ciencias Químicas y Farmacéuticas y Facultad de Medicina, Universidad de Chile. Santiago, Chile.

${ }^{6}$ Cardiology Division, Department of Internal Medicine, University of Texas Southwestern Medical Center, Dallas, Texas, USA. aBecado de Cardiología, Pontificia Universidad Católica de Chile. Santiago, Chile.

Este trabajo se financió con el apoyo del Proyecto FONDAP 15130011 (a AC, PFC y SL). Los autores declaran no tener conflictos de interés.

Recibido el 23 de noviembre de 2016, aceptado el 23 de mayo de 2017.

Correspondencia a: Drs. Gonzalo Martínez R. o Dr. Pablo Castro G. División de Enfermedades Cardiovasculares, Pontificia Universidad Católica de Chile. Marcoleta $367,2^{\circ}$ piso. gjmartin@uc.cl pcastro@med.puc.cl

\section{Cardiotoxicidad inducida por tratamientos oncológicos. Fundamentos para la implementación de equipos de Cardio-Oncología}

\author{
RENÉ HAMEAU ${ }^{1, a}$, LUIGI GABRIELLI ${ }^{1,2}$, \\ MARCELO GARRIDO ${ }^{3}$, ANA MARÍA GUZMÁN ${ }^{4}$, \\ IGNACIO RETAMAL ${ }^{3}$, MARÍA JOSÉ VACAREZZA', \\ DOUGLAS GREIG ${ }^{1}$, MAURICIO OCQUETEAU ${ }^{3}$, \\ CÉSAR SÁNCHEZ ${ }^{3}$, MARCELA PIZARRO ${ }^{5}$, \\ ALEJANDRO CORVALÁN ${ }^{2,3}$, SERGIO LAVANDERO ${ }^{5,6}$, \\ PABLO F. CASTRO ${ }^{1,2}$, GONZALO MARTÍNEZ ${ }^{1,2}$
}

\section{Cardiotoxicity of anticancer therapies. Towards the implementation of cardio-oncology units}

Recently, we have witnessed major improvements in cancer treatment. Early diagnosis and development of new therapies have reduced cancer-related mortality. However, these new therapies, along with greater patient survival, are associated with an increase in untoward effects, particularly in the cardiovascular system. Although cardiotoxicity induced by oncologic treatments affects predominantly the myocardium, it can also involve other structures of the cardiovascular system, becoming one of the main causes of morbidity and mortality in those who survive cancer. The main objective of cardio-oncology is to achieve the maximum benefits of oncologic treatments while minimizing their deleterious cardiovascular effects. It harbors the stratification of patients at risk of cardiotoxicity, the implementation of diagnostic tools (imaging techniques and biomarkers) for early diagnosis, preventive strategies and early treatment options for the complications. Herein, we discuss the basic knowledge for the implementation of cardio-oncology units and their role in the management of cancer patients, the diagnostic tools available to detect cardiotoxicity and the present therapeutic options.

(Rev Med Chile 2018; 146: 68-77)

Key words: Cardiotoxicity; Drug Therapy; Heart Failure; Cancer; Radiotherapy.

$\square$

urante las últimas décadas ha habido un aumento progresivo en las terapias disponibles para el tratamiento de los pacientes con enfermedades oncológicas, lo cual se ha acompañado de una mejoría significativa en la sobrevida de estos enfermos ${ }^{1}$. Sin embargo, muchas de las drogas antineoplásicas utilizadas, tal como la radioterapia torácica, tienen potenciales efectos dañinos sobre el sistema cardiovascular ${ }^{2}$. Adicionalmente, la patología cardiovascular es una de las principales fuentes de morbimortalidad en pacientes con enfermedades oncológicas ${ }^{3}$. Es importante reconocer que las enfermedades cardiovasculares (ECV) y los cánceres comparten factores de riesgo en común, tales como edad, estilo de vida no saludable, obesidad y tabaquismo ${ }^{4}$, 
que pueden determinar tanto la aparición como la progresión de ambos grupos de enfermedades. Por otro lado, es conocido el daño directo de ciertos tipos de neoplasias sobre el tejido cardiaco. Por ejemplo, $50 \%$ de los pacientes que desarrollan síndromes carcinoides tiene compromiso cardiaco ${ }^{5}$.

La estrecha interacción entre oncología y ECV ha determinado un creciente interés por establecer equipos dedicados a los cuidados cardiovasculares de los pacientes con cáncer ${ }^{6}$. Estos equipos proveen de cuidados clínicos a los pacientes previo al inicio de las terapias oncológicas, durante estas y en el seguimiento alejado tras una terapia exitosa. Además, han permitido expandir los conocimientos actuales sobre la epidemiología de la afectación cardiaca por distintas terapias oncológicas, los mecanismos involucrados en la cardiotoxicidad y los potenciales tratamientos para prevenir o tratar los efectos adversos.

En el presente artículo se discuten los fundamentos que sustentan el estudio de la cardiotoxicidad asociada a terapias oncológicas, las estrategias de prevención y tratamiento y el rol que pueden tener estas unidades en el país.

\section{Terapias oncológicas y cardiotoxicidad}

El volumen de pacientes sometidos a terapias oncológicas con resultado exitoso se ha incrementado dramáticamente en las últimas décadas ${ }^{7}$; por ejemplo, en linfomas, la sobrevida ha experimentado un aumento desde $47 \%$ en 1979 a $70 \%$ en 2010
(Tabla 1$)^{8}$. La mayor sobrevida de los enfermos ha hecho evidente la aparición de eventos cardiovasculares en el seguimiento y de efectos adversos a las drogas oncológicas 9 . Por ejemplo, las ECV dan cuenta de alrededor de la mitad de las causas de muerte en mujeres con cáncer de mama, en particular aquellas en etapas precoces, con mayor probabilidad de sobrevivir a su tumor ${ }^{10}$.

\section{Toxicidad por drogas oncológicas}

La lista de fármacos asociados a daño cardiovascular es extensa (Tabla 2), donde destacan las antraciclinas y las terapias biológicas. Dentro de estas últimas, trastuzumab ha sido la droga más estudiada ${ }^{11}$.

\section{A. Antraciclinas}

Estas drogas son responsables de la forma más conocida de cardiotoxicidad. Su incidencia es variable según la serie y el tipo de molécula utilizada: con doxorrubicina se describe una incidencia que puede llegar hasta $26 \%{ }^{12,13}$, la cual depende de algunos factores como la edad de los pacientes y la dosis total acumulada recibida, así como también si el desenlace reportado es disfunción ventricular o insuficiencia cardiaca clínica. El daño agudo por antraciclinas suele desarrollarse al momento de recibir la droga o dentro de la primera semana y asemeja el daño de una miocarditis aguda con injuria de los miocitos, infiltrados inflamatorios y edema intersticial ${ }^{14}$. Suele manifestarse como cambios electrocardiográficos o arritmias, elevación de

Tabla 1. Cánceres y sobrevida en 2 períodos de tiempo

\begin{tabular}{|cccc|}
\hline Subtipo de cáncer & Nuevos casos en $\mathbf{2 0 1 4}$ & $\begin{array}{c}\text { \% de sobrevida a } \\
\mathbf{5} \text { años en 1975-79 }\end{array}$ & $\begin{array}{c}\text { \% de sobrevida a } \\
\mathbf{5} \text { años en 2000-10 }\end{array}$ \\
\hline Próstata & 233.000 & 68,7 & 99,6 \\
\hline Mama & 232.670 & 74,6 & 90,6 \\
\hline Pulmón & 224.210 & 12,5 & 17,5 \\
\hline Colon & 136.830 & 50,4 & 65,9 \\
\hline Melanoma & 76.100 & 82 & 92,8 \\
\hline Vejiga & 74.690 & 73 & 80,6 \\
\hline Linfoma & 70.800 & 46,8 & 70,3 \\
\hline Leucemia & 52.380 & 34,6 & 60,8 \\
\hline Riñón & 63.920 & 50,9 & 73,5 \\
\hline Páncreas & 46.420 & 2,5 & 7,3 \\
\hline
\end{tabular}

Adaptado de Petek et cols ${ }^{8}$ 
Tabla 2. Terapia oncológicas y riesgo de toxicidad

\begin{tabular}{|c|c|c|}
\hline Terapia oncológica & Incidencia & Mecanismo propuesto \\
\hline $\begin{array}{l}\text { Antraciclinas } \\
\text { (doxorrubicina, epirrubicina, idarrubicina) }\end{array}$ & $3-26 \%$ & $\begin{array}{l}\text { Producción de radicales libres y aumento de estrés } \\
\text { oxidativo. Alteración de hebra de ADN asociada a } \\
\text { topoisomerasa-II-b }\end{array}$ \\
\hline $\begin{array}{l}\text { Agentes alquilantes } \\
\text { (ciclofosfamida, ifosfamida) }\end{array}$ & $7-28 \%$ & Aumento de radicales libres. Daño endotelial directo \\
\hline $\begin{array}{l}\text { Anticuerpos monoclonales } \\
\text { (trastuzumab, bevacizumab) }\end{array}$ & $2-28 \%$ & Inhibición de señalización de vía ERBB2 y VEGF \\
\hline $\begin{array}{l}\text { Antimetabolitos } \\
\text { (clofarabine, 5-fluouracilo, capecitabina, citarabina) }\end{array}$ & $2-27 \%$ & $\begin{array}{l}\text { No aclarado. Vasoespasmo, daño endotelial, daño } \\
\text { por metabolitos }\end{array}$ \\
\hline $\begin{array}{l}\text { Inhibidores de tirosina kinasa } \\
\text { (imatinib, dasatinib) }\end{array}$ & $2-4 \%$ & $\begin{array}{l}\text { Inhibición de Abl kinasa disfunción mitocondrial, } \\
\text { sobrecarga de volumen }\end{array}$ \\
\hline $\begin{array}{l}\text { Inhibidores multi-kinasa } \\
\text { (sunitinib) }\end{array}$ & $15-20 \%$ & Inhibición de kinasas e hipertensión arterial \\
\hline $\begin{array}{l}\text { Inhibidores de ERBB2 } \\
\text { (lapatinib) }\end{array}$ & $1,5-2,2 \%$ & $\begin{array}{l}\text { Inihibición de ERBB2 y receptor de factor de creci- } \\
\text { miento endotelial }\end{array}$ \\
\hline $\begin{array}{l}\text { Antimicrotúbulos } \\
\text { (docetaxel, paclitaxel) }\end{array}$ & $2,3-8 \%$ & Disfunción contráctil, daño organelos subcelulares \\
\hline $\begin{array}{l}\text { Inhibidores de proteosomas } \\
\text { (bortezomib, carfilzomib) }\end{array}$ & $2-5 \%$ & $\begin{array}{l}\text { Interferencia con sistema de ubiquitinas llevando a } \\
\text { acumulación de proteínas tóxicas }\end{array}$ \\
\hline
\end{tabular}

biomarcadores séricos y rara vez como disfunción ventricular ${ }^{15}$. También se ha descrito pericarditis aguda. En la forma crónica, el daño se manifiesta dentro del primer año o incluso más tardíamente, suele ser irreversible (o revertir parcialmente, según lo propuesto recientemente por Cardinale y $\operatorname{cols}^{16}$, alterando la ultraestructura tisular, y es dosis dependiente, habiéndose determinado que con dosis sobre $450 \mathrm{mg} / \mathrm{m}^{2}$ el riesgo aumenta significativamente ${ }^{17}$.

Existen múltiples mecanismos descritos de cardiotoxicidad por antraciclinas: a) generación de especies reactivas del oxígeno y de estrés oxidativo, generando daño a los ADNs genómico y mitocondrial ${ }^{18}$; b) desacoplamiento de la cadena transportadora de electrones y deterioro de la fosforilación oxidativa, perpetuando disfunción mitocondrial y daño celular; c) alteraciones en mecanismos de autofagia basal, como ha sido mostrado recientemente por nuestro grupo ${ }^{19} ; \mathrm{d}$ ) inhibición de topoisomerasa II-b, con la consiguiente desestabilización de la hebra de ADN, considerado en la actualidad uno de los efectos principales de estos medicamentos ${ }^{20}$.

\section{B. Trastuzumab}

Corresponde a un anticuerpo monoclonal que interfiere con el receptor HER2/ErbB2, presente en algunos cánceres ${ }^{21}$. Ensayos clínicos con trastuzumab reportan una incidencia de falla cardiaca clínica de 1,7-4,1\% y deterioro de la función ventricular izquierda de hasta $18,6 \%{ }^{22}$. A diferencia de las antraciclinas, el efecto suele ser reversible y no relacionado a dosis. El mecanismo responsable de cardiotoxicidad es la interferencia en la dimerización normal entre ErbB2 y ErbB4, lo que impide la activación de una vía fundamental para la sobrevida y adaptación de los miocardiocitos a situaciones de estrés celular ${ }^{23}$. A modo de ejemplo, ratones con deleciones específicas cardiacas de ErbB2 desarrollan miocardiopatía dilatada de manera espontánea y son incapaces de tolerar sobrecargas de presión o la administración concomitante de antraciclinas ${ }^{24}$. Estos hallazgos son refrendados por la experiencia clínica de que el riesgo de cardiotoxicidad por trastuzumab aumenta significativamente cuando se administra en asociación con antraciclinas ${ }^{25}$. 


\section{Terapias moleculares}

Estos fármacos, recientemente introducidos en el arsenal de terapias oncológicas, actúan bloqueando la acción del factor de crecimiento vascular endotelial (VEGF) y/o el factor de crecimiento derivado de plaquetas (PDGF), con lo cual inhiben la angiogénesis necesaria para el crecimiento de los tumores ${ }^{26}$. Este mecanismo de acción desestabiliza una serie de mecanismos de homeostasis, lo que se traduce en un aumento en la incidencia de hipertensión arterial, fenómenos vasoespásticos, isquemia miocárdica, disfunción sistólica y eventos tromboembólicos ${ }^{27}$. Dado su reciente introducción a la práctica clínica y la constante adopción de nuevas moléculas, el alcance de la cardiotoxicidad producida por estas drogas es aún incierto.

\section{Toxicidad por radioterapia}

El espectro de efectos cardiotóxicos de la radioterapia es extenso y puede afectar a todas las estructuras del corazón ${ }^{28}$. Dosis torácicas superiores a 30 Gy se asocian a efectos dañinos sobre el sistema cardiovascular, los cuales pueden aparecer muy tardíamente en la evolución de estos enfermos, incluso décadas después de recibida la radioterapia ${ }^{29}$. Pericarditis crónica se describe en $3 \%$ a 20 años y $12 \%$ a 30 años luego de irradiación superior a $35 \mathrm{~Gy}^{30}$. También se describen procesos fibróticos, tanto del endocardio como del aparato valvular que pueden causar valvulopatías ${ }^{31}$, daño microvascular coronario ${ }^{32}$ y mayor incidencia de enfermedad aterosclerótica, afectando típicamente los segmentos proximales del árbol coronario ${ }^{33}$. Por ejemplo, sobrevivientes de linfoma no Hodgkin tienen un incremento de 4-6 veces en el riesgo de desarrollar cardiopatía coronaria, comparado con la población general y en mujeres asintomáticas sometidas a radioterapia torácica hasta $50 \%$ presentan nuevos defectos de perfusión ${ }^{34}$.

Dado lo anterior, actualmente se sugiere un tamizaje de enfermedad coronaria a partir de los 10-15 años desde la terapia inicial y seguimiento de por vida ${ }^{35}$.

\section{Utilidad de la evaluación de pacientes sometidos a terapias oncológicas}

La evaluación y manejo cardiovascular de los enfermos sometidos a tratamientos oncológicos provee una serie de beneficios. Primero, una evaluación previa a la terapia permite categorizar el riesgo de cada paciente individual en cuanto a presentar una complicación cardiovascular ${ }^{36}$. Así, se pueden establecer estrategias específicas antes del inicio de la terapia oncológica, como preferir medicamentos con menor potencial cardiotóxico, ajustar los volúmenes de infusión a pacientes con disfunción sistólica o usar algunos medicamentos que podrían prevenir el daño en pacientes de alto riesgo ${ }^{37,38}$.

Segundo, la evaluación continua de estos pacientes durante la terapia oncológica puede permitir la detección precoz de daño miocárdico subclínico. Esto puede favorecer la reducción o el cambio en las drogas antineoplásicas o la instauración de terapias (por ejemplo enalapril) que permitan evitar la aparición de cardiotoxicidad ${ }^{39}$. Del mismo modo, el reconocimiento precoz de una caída en la fracción de eyección puede permitir la oportuna implementación de terapia para insuficiencia cardiaca, lo cual ha mostrado revertir, al menos parcialmente, el daño producido por antraciclinas ${ }^{16}$.

Tercero, el seguimiento a largo plazo de estos pacientes permite pesquisar y tratar una fuente importante de morbilidad alejada en este grupo. El daño miocárdico, coronario, valvular y pericárdico pueden aparecer en forma tardía, de modo que el seguimiento de pacientes seleccionados -según su riesgo- es necesario para asegurar una evolución alejada favorable ${ }^{40}$.

\section{Métodos para pesquisar cardiotoxicidad}

\section{A. Ecocardiografía}

La evaluación ecocardiográfica de la función ventricular izquierda es el pilar fundamental, tanto así que la definición misma de cardiotoxicidad incluye la caída de la fracción de eyección de ventrículo izquierdo (FEVI) (caída de $10 \%$ o a menos de $50 \%)^{41}$. Por ejemplo, el Canadian Trastuzumab Working Group recomienda monitorizar la FEVI al menos cada 3 meses hasta completar la terapia con trastuzumab ${ }^{42}$. El desarrollo de técnicas 3D ofrece beneficios adicionales para la estimación de la FEVI, considerándose la técnica ecocardiográfica con menor variabilidad intra e interobservador para la evaluación de la función ventricular en pacientes sometidos a quimioterapia. Sin embargo, esperar un deterioro en la función sistólica global medida por métodos volumétricos puede 
ser un marcador muy tardío de cardiotoxicidad ${ }^{43}$. En ese sentido, las técnicas ecocardiográficas de deformación de imagen (particularmente strain por speckle tracking) pudieran ser una alternativa útil para la detección más precoz de alteraciones en la motilidad del miocardio. Se describe que una caída en 10-15\% del strain longitudinal global del ventrículo izquierdo en 4 cámaras por speckle tracking es el parámetro más útil para la detección de cardiotoxicidad ${ }^{44}$ (Figura 1), sin embargo, se requieren más estudios para determinar el verdadero rol de esta nueva tecnología.

\section{B. Biomarcadores}

Ciertos biomarcadores séricos (troponinas, péptidos natriuréticos, proteína $\mathrm{C}$ reactiva, entre otros) pueden ser una herramienta complementaria a las técnicas de imágenes para la detección pre$\mathrm{coz}$ y el seguimiento de este grupo de pacientes. Su menor costo, mayor reproducibilidad (facilitando comparaciones en el tiempo) y la posibilidad de adelantarse al daño funcional necesario para ser pesquisado por los métodos de imagen son sus mayores ventajas.

Por ejemplo, NT-proBNP se eleva precozmente luego de quimioterapia en dosis altas ${ }^{45}$ y la persistencia de niveles elevados se ha relacionado con desarrollo de disfunción ventricular en pacientes tratados con antraciclinas ${ }^{46}$. En mujeres con cáncer de mama, aquellas sin incremento de troponina al término del tratamiento tuvieron una baja tasa de eventos cardiovasculares y sin deterioro significativo de la FEVI a 3 años $^{47}$. De manera opuesta, aquellos pacientes que presentan niveles persistentemente elevados de troponina I durante el tratamiento presentan una menor FEVI final, asociado a mayores tasas de eventos

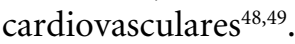

El uso de nuevas técnicas de imagen y biomarcadores, capaces de identificar daño miocárdico antes de que caiga la FEVI, han modificado nuestra comprensión y la forma de reconocer el daño por cardiotoxicidad. Es posible que en los próximos años la definición de cardiotoxicidad basada en la caída de la FEVI se modifique, buscando considerar estos nuevos parámetros más sensibles.

\section{Estrategias de prevención y manejo}

El principal rol de los equipos de Cardio-Oncología es prevenir el desarrollo de cardiotoxicidad. En aquellos pacientes con riesgo moderado o alto de presentar daño miocárdico se pueden implementar estrategias que reduzcan la exposición a cardiotóxicos o el inicio de fármacos cardioprotectores. A continuación mencionaremos las estrategias más comunes:

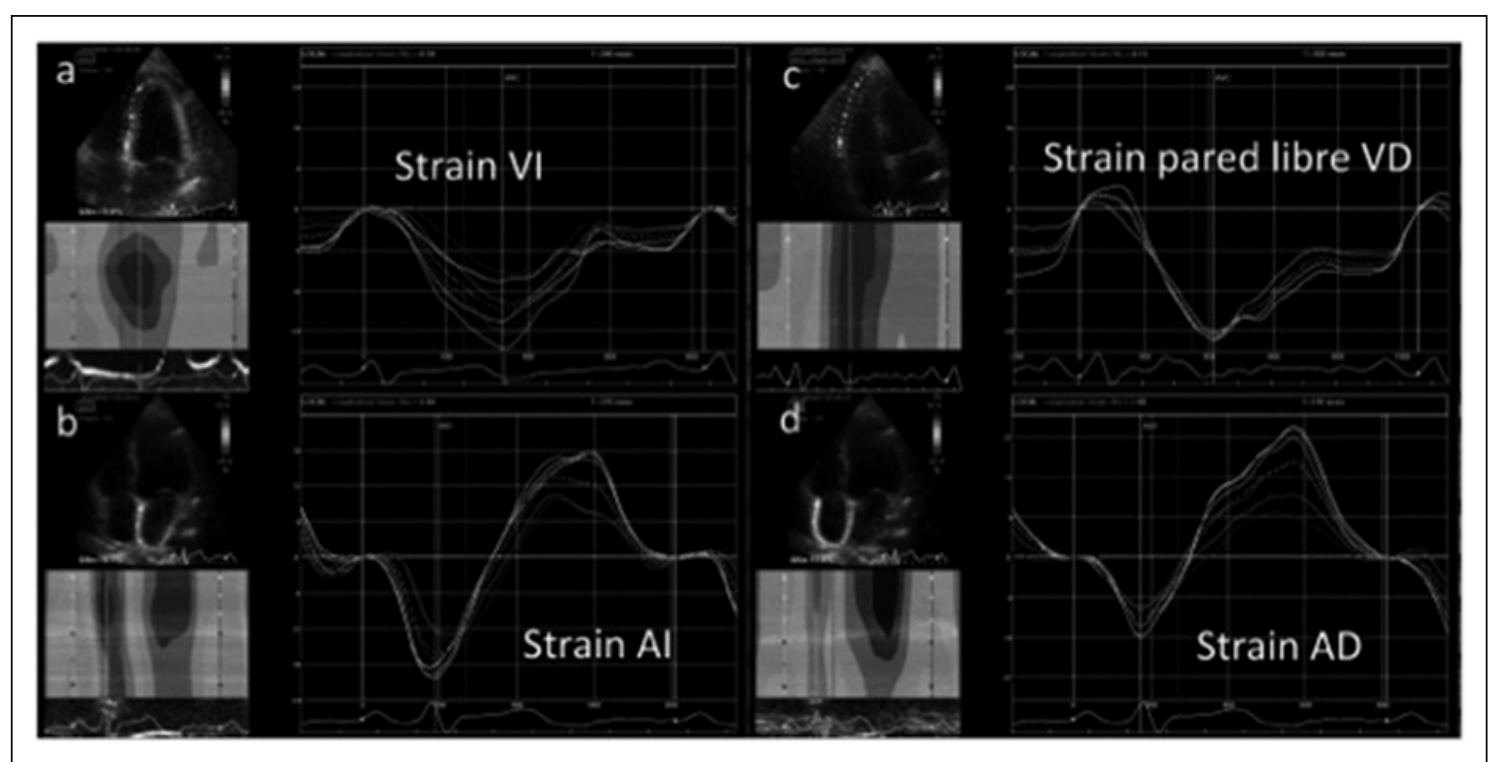

Figura 1. Técnicas de deformación. Curvas de strain que muestran la deformación durante el ciclo cardíaco en las 4 cavidades cardíacas: a) ventrículo izquierdo; b) aurícula izquierda; c) ventrículo derecho; d) aurícula derecha. 


\section{Modificación de la terapia oncológica}

La toxicidad por antraciclinas se relaciona con la dosis total acumulada, que en el caso de doxorrubicina no debiera superar los $450 \mathrm{mg} / \mathrm{m}^{2}$ en adultos. Reducir la dosis total de exposición a estas drogas disminuye su potencial efecto cardiotóxico. También, estrategias de infusión prolongada pueden reducir la incidencia de cardiotoxicidad comparado con esquemas en bolo ${ }^{50}$. Adicionalmente, la sustitución por fármacos menos cardiotóxicos (por ejemplo, epirrubicina) o la utilización de formulaciones liposomales pueden reducir aun más la tasa de efectos adversos cardiacos ${ }^{51}$. Otras estrategias implican la suspensión temporal o definitiva de la terapia cuando ocurre una caída de la FEVI; esto está particularmente validada para el daño reversible por trastuzumab.

\section{Fármacos cardioprotectores}

Numerosos estudios han evaluado el rol de la terapia estándar de insuficiencia cardiaca para la prevención y tratamiento de cardiotoxicidad. Otros agentes adicionales, como dexrazoxane, también han sido probados para limitar el daño por antraciclinas y trastuzumab ${ }^{52}$.

\section{A. $\beta$-Bloqueadores}

El carvedilol, un $\beta$-bloqueador con propiedades antioxidantes y quelantes de fierro, ha demostrado su utilidad como cardioprotector en pacientes que reciben antraciclinas. Kalay y colaboradores ${ }^{38}$ distribuyeron en forma aleatoria a 50 pacientes en quimioterapia con antraciclinas a recibir carvedilol 12,5 mg o placebo. Tras 6 meses, los pacientes del grupo control tuvieron una FEVI significativamente más baja, así como diámetros sistólicos y diastólicos del ventrículo izquierdo incrementados, en comparación al grupo que recibió carvedilol. Nebivolol, un $\beta$-bloqueador selectivo con capacidad liberadora de óxido nítrico, administrado a mujeres con cáncer de mama que recibieron antraciclinas, logró reducir las alteraciones en NT-pro-BNP y FEVI al compararlo con placebo ${ }^{53}$. Sin embargo, el efecto al parecer no es de clase, ya que algunas drogas (por ejemplo metoprolol) no han demostrado ser beneficiosas ${ }^{54}$.

\section{B. Inhibidores de la enzima convertidora de angiotensina (IECA)}

El efecto cardioprotector de enalapril fue evaluado en un estudio que incluyó 473 pacientes
(53\% con cáncer de mama) tratados con antraciclinas en altas dosis ${ }^{39}$. Ciento catorce pacientes (24\%) presentaron un incremento precoz de troponina y fueron distribuidos en forma aleatoria a recibir enalapril versus placebo. El grupo que recibió enalapril no mostró modificaciones significativas de la FEVI, mientras que el grupo placebo mostró una caída significativa, asociado a un incremento de los diámetros ventriculares. Este estudio, por lo tanto, apoya el uso de troponina como predictor precoz de daño $y$, a su vez, el beneficio de enalapril para minimizar el impacto de la terapia con antraciclinas. Se ha postulado que la combinación IECA y $\beta$-bloqueadores puede proveer un mayor beneficio. El estudio OVERCOME mostró que, en pacientes con neoplasias hematológicas sometidos a esquemas de quimioterapia intensiva, el uso de enalapril más carvedilol previno la caída de FEVI y redujo el desenlace clínico de muerte e insuficiencia cardia$\mathrm{Ca}^{37}$. En 201 pacientes con disfunción ventricular izquierda secundario al uso de antracilinas, se observó una relación significativa entre el inicio precoz de la terapia con enalapril + carvedilol y la normalización de la función ventricular (FEVI $>50 \%$ ), poniendo énfasis en la importancia del tiempo en la instauración de la terapia ${ }^{55}$.

\section{Estatinas}

Los efectos pleiotrópicos (antioxidantes y antiinflamatorios) de las estatinas pudieran tener un potencial rol cardioprotector. En un modelo animal, fluvastatina redujo el estrés oxidativo por antraciclinas al aumentar la expresión de superóxido-dismutasa mitocondrial, limitando la inflamación cardiaca ${ }^{56}$. Estudios observacionales sugieren que estatinas se asocian a una reducción del riesgo de falla cardiaca en mujeres con cáncer de mama tratadas con antraciclinas ${ }^{57}$. Si bien su uso parece promisorio, actualmente no contamos con evidencia definitiva que apoye su prescripción en este escenario.

\section{Dexrazoxane}

Es un quelante del hierro EDTA-símil, que se une a este y reduce la formación de radicales superóxido causantes de daño oxidativo miocárdico. Además, pareciera tener un efecto sobre la estabilidad de las hebras de ADN a nivel cardiaco, promoviendo la acción de topoisomerasa-II-b. Su eficacia ha sido apoyada por una reciente revisión 
Cochrane $e^{58}$, siendo recomendada actualmente para pacientes con cáncer de mama metastásico que hayan recibido una dosis acumulada de doxorrubicina $>300 \mathrm{mg} / \mathrm{m}^{2(59)}$. Estudios recientes también sugieren su utilidad en pacientes con cáncer de mama HER-2 positivo ${ }^{60}$. Su uso no se ha masificado, principalmente por cuestionamientos respecto de que pudiera disminuir la eficacia antineoplásica de las antraciclinas, ocurrencia de neoplasias secundarias o posibles efectos aditivos mielosupresores. A pesar de ello, aún es considerada como una alternativa preventiva por las actuales guías europeas ${ }^{35}$.

\section{Funcionamiento de unidades de Cardio-Oncología}

De acuerdo a lo expuesto previamente, es importante el desarrollo de equipos de trabajo especializados en los cuidados de estos pacientes. En nuestra opinión, las estructuras habitualmente presentes en los departamentos de Cardiología, tales como laboratorios de ecocardiografía y programas de insuficiencia cardiaca y trasplante, pueden no cubrir adecuadamente las necesidades propias de estos pacientes complejos.

Nuestro grupo ha implementado una unidad de Cardio-Oncología multidisciplinaria, con la participación activa de integrantes de ambas espe- cialidades, además de enfermeras exclusivas, especialistas en laboratorio clínico y coordinadores de investigación (Figura 2). Esta unidad se preocupa de la categorización de riesgo e implementación de estrategias preventivas en pacientes sometidos a terapias oncológicas, del tratamiento especializado de pacientes que han sufrido cardiotoxicidad y del seguimiento alejado de pacientes con un perfil de riesgo aumentado que han recibido terapias oncológicas, radioterapia o ambos. Las intervenciones y protocolos de seguimiento han sido estandarizados, para así facilitar la interacción entre los distintos especialistas y son coordinados por la enfermera a cargo de la unidad. Adicionalmente, nuestro programa considera la realización de investigación básica, preclínica y clínica, para lo cual cuenta con un registro de datos prospectivo, la creación de un biobanco para almacenar muestras de manera seriada y la integración con centros de investigación biomédica. Finalmente, cuenta con reuniones periódicas entre los especialistas para coordinar el funcionamiento del programa, discutir aquellos casos más complejos, optimizar protocolos y definir estrategias de investigación.

\section{Conclusiones}

Las complicaciones cardiovasculares de las terapias antineoplásicas constituyen un elemento que deteriora la calidad de vida y empeora el

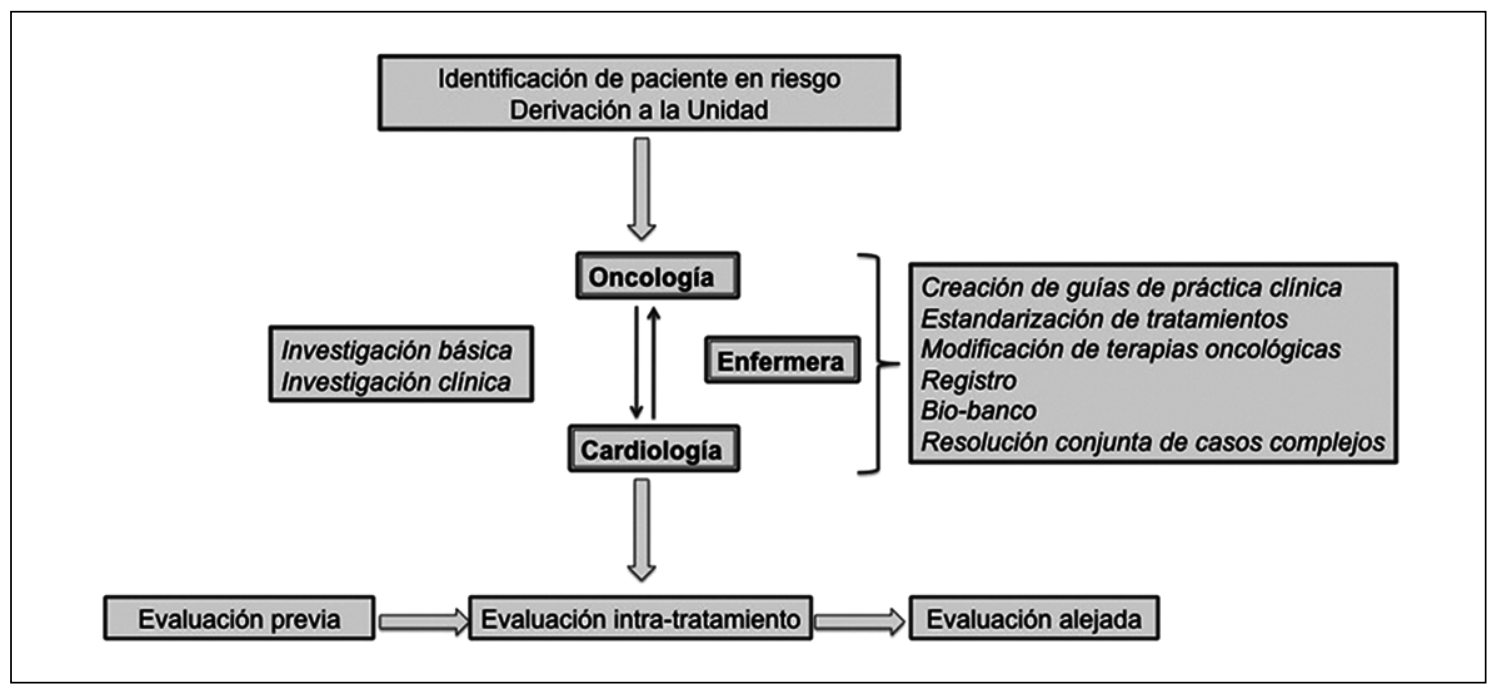

Figura 2. Funcionamiento de unidad de Cardio-Oncología UC. La unidad de Cardio-Oncología de nuestra institución se basa en la estrecha relación entre cardiólogos y oncólogos. Sus acciones incluyen cuidados clínicos de los pacientes, generación de protocolos de tratamiento y generación de investigación. 


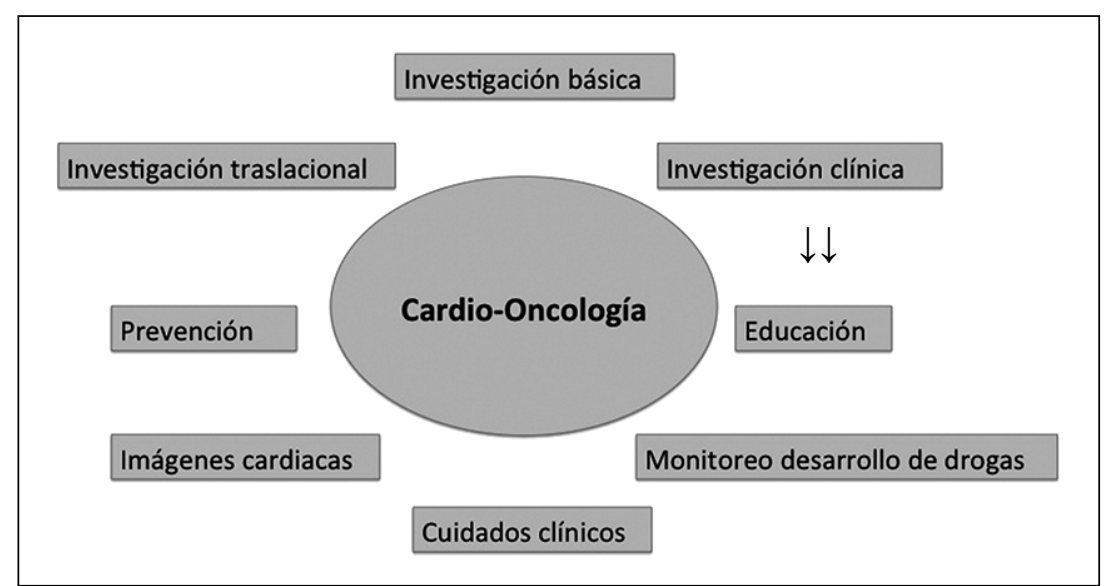

Figura 3. Rol de las unidades de Cardio-Oncología. La interacción multidisciplinaria clínica y básica es fundamental para el desarrollo de esta especialidad. pronóstico de los pacientes oncológicos. Afortunadamente, el abordaje multidisciplinario permite implementar estrategias para prevenir, identificar y tratar precoz y eficazmente estas complicaciones. La pesquisa mediante el uso de técnicas de imagen y biomarcadores nos permiten identificar incluso aquellos pacientes con signos subclínicos de cardiotoxicidad.

A futuro, mayores estudios clínicos debieran clarificar la utilidad de la terapia preventiva con $\beta$-bloqueadores e IECA, así como de otras moléculas potencialmente cardioprotectoras o el uso de nuevos esquemas oncológicos que reduzcan el daño cardiovascular.

Existe la necesidad de colaboración entre cardiólogos, oncólogos y otros profesionales para la elaboración en conjunto de protocolos de tratamiento, programas de educación y el desarrollo de nueva evidencia científica que aborde de forma integral el cuidado de los pacientes oncológicos y permita generar nuevo conocimiento en este floreciente campo.

\section{Referencias}

1. Siegel R, Naishadham D, Jemal A. Cancer statistics for Hispanics/Latinos. CA Cancer J Clin 2012; 62: 283-98.

2. Dent S, Liu P, Brezden-Masley C, Lenihan D. Cancer and Cardiovascular Disease: The Complex Labyrinth. J Oncol 2015; 2015: 516450.

3. Siegel R, De Santis C, Virgo K, et al. Cancer treatment and survivorship statistics. CA Cancer J Clin 2012; 62: 220-41.

4. Johnson CB, Davis MK, Law K, Suplher J. Shared risk factors for cardiovascular disease and cancer: Implications for preventive health and clinical care in oncology patients. Can J Cardiol 2016; 32: 900-7.

5. Paredes A, Valdebenito M, Neira V, Castro PF, Zalaquett R. Síndrome carcinoide y compromiso valvular cardíaco. Rev Chil Cardiol 2014; 33: 38-43.

6. Herrmann J, Lerman A, Sandhu NP, Villarraga HR, Mulvagh SL. Evaluation and management of patients with heart disease and cancer: cardio-oncology. Mayo Clin Proc 2014; 89 (9): 1287-306.

7. Howlader N, Ries LA, Mariotto AB, Reichmann ME, Ruhl J, Cronin KA. Improved estimates of cancer-specific survival rates from population-based data. J Natl Cancer Inst 2010; 102 (20): 1584-98.

8. Petek BJ, Greenman C, Herrmann J, Ewer MS, Jones RL. Cardio-oncology: an ongoing evolution. Future Oncol 2015; 11 (14): 2059-66.

9. Aleman BM, Moser EC, Nuver J, Suter TM, Maraldo MV, Specht L, et al. Cardiovascular disease after cancer therapy, EJC Suppl 2014; 12: 18-28.

10. Patnaik JL, Byers T, Di Guiseppi C, Dabelea D, Denberg TD. Cardiovascular disease competes with breast cancer as the leading cause of death for older females diagnosed with breast cancer: a retrospective cohort study. Breast Cancer Res 2011; 13 (3): R64.

11. Curigliano G, Cardinale D, Dent S, Criscitiello C, Aseyev $\mathrm{O}$, Lenihan D, et al. Cardiotoxicity of anticancer treatments: Epidemiology, detection, and management. CA: Cancer J Clin 2016; 66 (4): 309-25.

12. Smith LA, Cornelius VR, Plummer CJ, Levitt G, Verrill $\mathrm{M}$, Canney $\mathrm{P}$, et al. Cardiotoxicity of anthracycline agents for the treatment of cancer: systematic review and meta-analysis of randomised controlled trials. BMC cancer 2010; 10: 337-39. 
13. Zhang S, Liu X, Bawa-Khalfe T, Lu LS, Liu LF. Identification of the molecular basis of doxorubicin-induced cardiotoxicity. Nat Med 2012; 18: 1639-42.

14. Ferrans VJ. Overview of cardiac pathology in relation to anthracycline cardiotoxicity. Cancer Treat Rep 1978; 62 (6): 955-61.

15. Shan K, Lincoff AM, Young JB. Anthracycline-induced cardiotoxicity. Ann Intern Med 1996; 125 (1): 47-58.

16. Cardinale D, Colombo A, Bacchiani G, Tedeschi I, Meroni CA, Veglia F, et al. Early detection of anthracycline cardiotoxicity and improvement with heart failure therapy. Circulation 2015; 131 (22): 1981-8.

17. Volkova M, Russell R. Antracycline cardiotoxicity: prevalence, pathogenesis and treatment. Curr Cardiol Rev 2011; 7 (4): 214-20.

18. Yang F, Teves S, Kemp CJ, Henikoff S. Doxorubicin, DNA torsion, and chromatin dynamics. Biochim Biophys Acta 2014; 1845 (1): 84-9.

19. Pizarro M, Troncoso M, Martínez GJ, Chiong M, Castro PF, Lavandero S. Basal autophagy protects cardiomyocytes from doxorubicin induced toxicity. Toxicology 2016; 370: 41-8.

20. Ky B, Vejpongsa P, Yeh ET, Force T, Moslehi JJ. Emerging paradigms in cardiomyopathies associated with cancer therapies. Circ Res 2013; 113: 754-64.

21. Hudis CA. Trastuzumab-mechanism of action and use in clinical practice. N Eng J Med 2007; 357 (1): 39-51.

22. Seidman, A, Hudis C, Pierri MK, Shak S, Paton V, Ashby $\mathrm{M}$, Cardiac dysfunction in the trastuzumab clinical trials experience. J Clin Oncol 2002; 20 (5): 1215-21.

23. Di Cosimo S. Heart to heart with trastuzumab: a review on cardiac toxicity. Target Oncology 2011; 6 (4): 189-95.

24. Crone SA, Zhao YY, Fan L, Gu Y, Minamisawa S, et al. ErbB2 is essential in the prevention of dilated cardiomyopathy. Nat Med 2002; 8 (5): 459-65.

25. Xue J, Iang Z, Qi F, Lv S, Zhang S, Wang T. Risk of trastuzumab-related cardiotoxicity in early breast cancer patients: a prospective observational study. J Breast Cancer 2014; 17 (4): 363-9.

26. Force T, Kolaja KL. Cardiotoxicity of kinase inhibitors: the prediction and translation of preclinical models to clinical outcomes. Nat Rev Drug Discov 2011; 10 (2): 111-26.

27. Cheng H, Force T. Molecular Mechanisms of Cardiovascular Toxicity of Targeted Cancer Therapeutics. Circ Res 2010; 106 (1): 21-34.

28. Eldabaje R, Le DL, Huang W, Yang LX. Radiation-associated Cardiac Injury. Anticancer Res 2015; 35 (5): 2487-92.

29. Stewart JR, Fajardo LF, Gillette SM, Constine LS. Radiation injury to the heart. Int J Radiat Oncol Biol Phys
1995; 31 (5): 1205-11.

30. Mulrooney D, Yeazel M, Kawashima T, Mertens AC, Mitby P, Stovall M, et al. Cardiac outcomes in a cohort of adult survivors of childhood and adolescent cancer: retrospective analysis of the Childhood Cancer Survivor Study cohort. BMJ 2009; 339: b4606.

31. Ong DS, Aertker RA, Clark AN, Kiefer T, Hughes GC, Harrison JK. Radiation-associated valvular heart disease. J Heart Valve Dis 2013; 22 (6): 883-92.

32. Veinot JP, Edwards WD. Pathology of radiation-induced heart disease: a surgical and autopsy study of 27 cases. Hum Pathol 1996; 27 (8): 766-73.

33. Heidenreich PA, Schnittger I, Strauss HW. Screening for coronary artery disease after mediastinal irradiation for Hodgkin's disease. J Clin Onc 2007; 25 (1): 43-9.

34. Yusuf SW, Sami S, Daher IN. Radiation-induced heart disease: a clinical update. Cardiol Res Pract 2011; 2011 (4): 317659.

35. Zamorano JL, Lancellotti P, Muñoz DR, Aboyans V, Asteggiano R, Galderisi M. ESC Position Paper on cancer treatments and cardiovascular toxicity developed under the auspices of the ESC Committee for Practice Guidelines: The Task Force for cancer treatments and cardiovascular toxicity of the European Society of Cardiology (ESC). Eur Heart J 2016; 37 (36): 2768-801.

36. Johnson CB, Sulpher J, Stadnick E. Evaluation, prevention and management of cancer therapy-induced cardiotoxicity: a contemporary approach for clinicians. Curr Opin Cardiol 2015; 15: 197-204.

37. Bosch X, Rovira M, Sitges M, Domenech A, Ortiz-Pérez JT, de Carralt TM, et al. Enalapril and carvedilol for preventing chemotherapy-induced left ventricular systolic dysfunction in patients with malignant hemopathies: the OVERCOME trial (prevention of left ventricular dysfunction with enalapril and carvedilol in patients submitted to intensive Chem Otherapy for the treatment of malignant hemopathies). J Am Coll Cardiol 2013; 61 (23): 2355-62.

38. Kalay N, Basar E, Ozdogru I, Er O, Cetinkaya Y, Dogan A, et al. Protective effects of carvedilol against anthracycline-induced cardiomyopathy. J Am Coll Cardiol 2006; 48 (11): 2258-62.

39. Cardinale D, Colombo A, Sandri MT, Lamantia G, Colombo N, Civelli M, et al. Prevention of high-dose chemotherapy induced cardiotoxicity in high-risk patients by angiotensin-converting enzyme inhibition. Circulation 2006; 114 (23): 2474-81.

40. Lenneman CG, Sawyer DB. Cardio-Oncology: An Update on Cardiotoxicity of Cancer-Related Treatment. Circ Res 2016; 118 (6): 1008-20.

41. Bloom MW, Hamo CE, Cardinale D, Ky B, Nohria A, 
Baer L, et al. Cancer Therapy-Related Cardiac Dysfunction and Heart Failure: Part 1: Definitions, Pathophysiology, Risk Factors, and Imaging. Circ Heart Fail 2016; (9): e002661.

42. Mackey JR, Clemons M, Cote MA, Delgado D, Dent S, Paterson A y cols. Cardiac management during adjuvant trastuzumab therapy: recommendations of the Canadian Trastuzumab Working Group. Curr Oncol 2008; 15 (1): 24-35.

43. Thavendiranathan $\mathrm{P}$, Grant AD, Negishi T, Plana JC, Popovic ZB, Marwick TH. Reproducibility of echocardiographic techniques for sequential assessment of left ventricular ejection fraction and volumes: application to patients undergoing cancer chemotherapy. J Am Coll Cardiol 2013; 61 (1): 77-84.

44. Rhea IB, Uppuluri S, Sawada S, Schneider BP, Feigenbaum $H$. Incremental prognostic value of echocardiographic strain and its association with mortality in cancer patients. J Am Soc Echocardiogr 2015; 28 (6): 667-73.

45. Sandri MT, Salvatici M, Cardinale D, et al. N-terminal pro-B-type natriuretic peptide after high-dose chemotherapy: a marker predictive of cardiac dysfunction? Clin Chem 2005; 51 (8): 1405-10.

46. Romano S, Fratini S, Ricevuto E, et al. Serial measurements of NT-proBNP are predictive of not-high-dose anthracycline cardiotoxicity in breast cancer patients. Br J Cancer 2011; 105 (11): 1663-8.

47. Cardinale D, Sandri MT, Colombo A, Colombo A, Colombo N, Boeri M, et al. Prognostic value of troponin I in cardiac risk stratification of cancer patients undergoing high-dose chemotherapy. Circ 2004; 109 (22): 2749-54.

48. Katsurada K, Ichida M, Sakuragi M, et al. High-sensitivity troponin $\mathrm{T}$ as a marker to predict cardiotoxicity in breast cancer patients with adjuvant trastuzumab therapy. Springerplus 2014; 3: 620.

49. Grover S, Leong DP, Chakrabarty A, et al. Left and right ventricular effects of anthracycline and trastuzumab chemotherapy: a prospective study using novel cardiac imaging and biochemical markers. Int J Cardiol 2013; 168 (6): 5465-7.

50. Van Dalen EC, van der Pal HJ, Caron HN, Kremer LC. Different dosage schedules for reducing cardiotoxicity in cancer patients receiving anthracycline chemotherapy. Cochrane Database Syst Rev. 2016;4:CD005008. Disponible en:http://onlinelibrary. wiley.com/doi/10.1002/14651858.CD005008.pub4/full (consultado el 10 de diciembre de 2016).
51. Floyd J, Morgan JP. Cardiotoxicity of anthracycline-like chemotherapy agents. Basow DS, ed. UpToDate. 2016. Disponible en: https://www.uptodate.com/contents/cardiotoxicity-of-anthracycline-like-chemotherapy-agents/abstract/53 (consultado el 10 de diciembre de 2016).

52. Schuler MK, Gerdes S, West A, Richter S, Busemann C, Hentschel L, et al. Efficacy and safety of Dexrazoxane (DRZ) in sarcoma patients receiving high cumulative doses of anthracycline therapy-a retrospective study including 32 patients. Br Med Cl Cancer 2016; 16: 619.

53. Kaya MG, Ozkan M, Gunebakmaz O, Akkaya H, Kaya EG, Akpek M, et al. Protective effects of nebivolol against anthracycline-induced cardiomyopathy: a randomized control study. Int J Cardiol 2013; 167 (5): 2306-10.

54. Georgakopoulos P, Roussou P, Matsakas E, Karavidas A. Cardioprotective effect of metoprolol and enalapril in doxorubicin-treated lymphoma patients: a prospective, parallel-group, randomized, controlled study with 36-month follow-up. Am J Hematol 2010; 85 (11): 894-6.

55. Cardinale D, Colombo A, Lamantia G, Colombo N, Civelli M, De Giacomi G, et al. Anthracycline-Induced Cardiomyopathy: clinical relevance and response to pharmacologic therapy. J Am Coll Cardiol 2010; 55 (3): 213-20.

56. Riad A, Bien S, Westermann D, Becher PM, Loya K, Landmesser U, et al. Pretreatment with statin attenuates the cardiotoxicity of doxorubicin in mice. Cancer Res 2009; 69 (2): 695-99.

57. Seicean S, Seicean A, Plana JC, Budd GT, Marwick TH. Effect of statin therapy on the risk for incident heart failure in patients with breast cancer receiving anthracycline chemotherapy: an observational clinical cohort study. J Am Coll Cardiol 2012; 60 (23): 2384-90.

58. Van Dalen E, Caron H, Dickinson HO, Kremer LC. Cardioprotective interventions for cancer patients receiving anthracyclines. Cochrane Database Syst Rev 2008; (2): CD003917.

59. Hensley ML, Hagerty KL, Kewalramani T, Green DM, Meropol NJ, Wasserman TH, et al. American Society of Clinical Oncology 2008 clinical practice guideline update: use of chemotherapy and radiation therapy protectants. J Clin Oncol 2009; 27 (1): 127-45.

60. Kim IH, Lee JE, Youn HJ, Song BJ, Chae BJ. Cardioprotective Effect of Dexrazoxane in Patients with HER2-Positive Breast Cancer Who Receive Anthracycline Based Adjuvant Chemotherapy Followed by Trastuzumab. J Breast Cancer 2017; 20 (1): 82-90. 